\title{
THE CLASSIFICATION OF SIMPLY CONNECTED $H$-SPACES WITH THREE CELLS I
}

\section{A. ZABRODSKY}

\section{Introduction.}

Recently the list of known $H$-spaces with few cells was considerably enriched see [2], [4], [7] and [8]. Most of the non-classical $H$-spaces newly discovered were principal $G$-bundles over spheres where $G$ is a classical Lie group. In order to regain control of this new flow of $H$ spaces it seems desirable to obtain some necessary or sufficient conditions for $G$-bundles over spheres to be $H$-spaces. The following seems highly probable:

0.1. ConJecture. Let $\left(G_{n}, d\right)=(S U(n), 2)$ or $(S p(n), 4)$. Then it is well-known that

$$
\pi_{d n-2}\left(G_{n-1}\right)=\mathrm{Z}_{m}, \quad m=2^{r}(2 k+1), r>0 .
$$

Let $M(n, \lambda)$ be the total space of the following induced principal fibration:

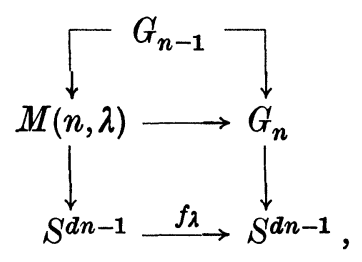

$\operatorname{deg} f_{\lambda}=\lambda . \quad\left(\right.$ In [7] and in the sequel, $M(2, \lambda)$ is denoted by $M_{\lambda}^{10}$ for $d=4$.)

0.1.1. If $(\lambda, m)=1$, then $M(n, \lambda)$ is a loop space. $M(n, \lambda) \approx M\left(n, \lambda^{\prime}\right)$ if and only if $\lambda \equiv \pm \lambda^{\prime}(\bmod m)$. For $d=4$ and $n=2$ one has $m=12, M(2,1)=$ $S p(2)$, and $M_{5}^{10}=M(2,5) \approx M(2,7)$ is the Hilton-Roitberg manifold proved to be a loop space by Stasheff [7].

0.1.2. If $\lambda$ is odd and satisfies: $p \mid \lambda$ and $p^{r} \mid m$ implies $p^{r} \mid \lambda$ for every prime $p$, then $M(n, \lambda)$ is an $H$-space. If $d n-1=7$, the restriction that $\lambda$ is odd is not necessary.

0.1.3. If $d n-1>7$ and $M(n, \lambda)$ admits an $H$-structure, then $\lambda$ is odd.

Received June 8, 1970.

Math. Scand. $30-13$ 
This paper is first in a sequence of two referred to as CSCH3 I and CSCH3 II. In CSCH3 II, conjectures 0.1.1 and 0.1.2 are given some consideration and an outline of a proof is given. In the case $d n-1<7$, conjecture 0.1.3 is very simple. As a whole 0.1 is verified in [2] for the case $d=2, d n-1=7$. The main object of CSCH3 $\mathrm{I}$ is to settle the case $d=4, d n-1=7$. It turns out that the validity of 0.1 .3 for $d=4, n=2$ was the last obstruction to the classification of simply connected $H$-spaces with three cells. This was also realized independently by Hilton-Roitberg [5] and by M. Curtis-Mislin-E. Thomas (Private communications). Thus, the ultimate goal of the present papers is to prove

0.2. The Classification theorem. Let $X$ be a simply connected $C W$ complex with three cells. If $X$ admits an $H$-structure, then $X$ is homotopy equivalent to one of the following eight complexes:

$$
S^{3} \times S^{3}, \quad S U(3), \quad M_{k}{ }^{10}, k=0,1,3,4,5, \quad S^{7} \times S^{7} .
$$

This theorem is proved in CSCH3 II. The main theorem in CSCH3 I is the following:

0.3. Matn Theorem I. Let $X$ be a simply connected $C W$ complex satisfying:

$$
\begin{aligned}
& H^{*}\left(X, \mathrm{Z}_{2}\right)=\Lambda\left(x_{3}, x_{7}\right) \text { in } \operatorname{dim} \leqq 13, x_{i} \in H^{i}\left(X, \mathrm{Z}_{2}\right), \\
& \quad{ }^{2} \pi_{6}(X)=\mathrm{Z}_{2} .
\end{aligned}
$$

If $X$ admits an $H$-structure, then $H^{14}\left(X, \mathrm{Z}_{2}\right) \neq 0$. In particular, the complexes $M_{k}{ }^{10}, k \equiv 2 \bmod 4$, do not admit $H$-structures.

The proof of 0.3 is based on calculations involving high order cohomology operations carried out in chapter 2. The proof is quite complex as the obstruction for $M_{k}^{10}$ to be an $H$-space can be essentially detected by a cohomology operation of order 5. Fortunately this operation can be decomposed in such a way that the calculations only involve operations of order at most three.

More precisely: Two operations $\varphi$ and $\bar{\varphi}$ are studied. Both are operations defined on $H^{*}\left(\cdot, \mathrm{Z}_{4}\right)$ classes with values being cosets of $H^{*}\left(\cdot, \mathrm{Z}_{2}\right)$ (referred to as $Z_{4}-Z_{2}$ operations). The operation $\varphi$ is a third-order operation of degree 8 , while $\bar{\varphi}$ is a secondary operation of degree 4 . The following type of relation between them is established (Proposition 2.3):

$$
\varphi 2 \supset S q^{4} \bar{\varphi}+S q^{8} \varrho_{2},
$$

where $\varrho_{2}$ is the reduction $H^{*}\left(\cdot, \mathbf{Z}_{4}\right) \rightarrow H^{*}\left(\cdot, \mathbf{Z}_{2}\right)$. The evaluations of $\varphi$ and $\bar{\varphi}$ on the projective plane $B_{2}(\hat{X})$ of $\hat{X}$ (where $\hat{X}$ is essentially $X$ made 4-connected) imply the condition $H^{14}\left(X, Z_{2}\right) \neq 0$. 


\section{Some definitions and notations.}

Let $I=\left(n_{1}, n_{2}, \ldots, n_{k}\right)$ be a finite sequence of natural numbers. Put $k=l(I)$ and write $K\left(\mathrm{Z}_{p}, I\right)$ for the product $\Pi_{j=1}^{k} K\left(\mathrm{Z}_{p}, n_{j}\right)$. The vector in $H^{*}\left(K\left(\mathrm{Z}_{p}, I\right), \mathrm{Z}_{p}\right)$ consisting of the images of the fundamental classes of $H^{n_{j}}\left(K\left(\mathrm{Z}_{p}, n_{j}\right), \mathrm{Z}_{p}\right)$ will be referred to as the fundamental vector.

If $I_{1}$ and $I_{2}$ are two sequences, an $H$-mapping $h$ (and hence $\infty$-loop map) between $K\left(Z_{p}, I_{1}\right)$ and $K\left(Z_{p}, I_{2}\right)$ can be given by an $l\left(I_{2}\right) \times l\left(I_{1}\right)$ matrix $B$ with entries in the Steenrod algebra $a(p): h^{*} \iota_{2}=B \iota_{1}$, where the $\iota_{k}$ are the fundamental vectors.

A (stable) generalized $k$-stage Postnikov system $(\bmod p)$ is an $\nsim$ deloopable diagram $(\mathscr{G})$ of the form

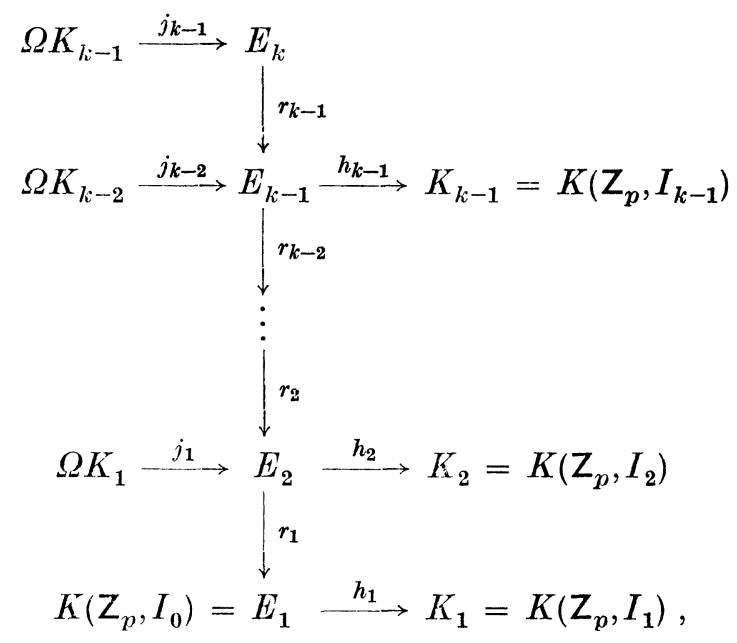

where

$$
\Omega K_{i-1} \stackrel{j_{i-1}}{\longrightarrow} E_{i} \stackrel{r_{i-1}}{\longrightarrow} E_{i-1}
$$

is the principal $\Omega K_{i-1}$ fibration induced by $h_{i-1}$. Let $B_{i}$ be the $l\left(I_{i}\right) \times l\left(I_{i-1}\right)$ matrix with entries in $a(p)$ corresponding to $h_{1}$ if $i=1$ and to $h_{i} \circ j_{i-1}$ if $k>i>1$. We refer to $(\mathscr{G})$ as to the geometric realization of $B_{1}, \ldots, B_{k-1}$. Conversely, given $B_{1}, \ldots, B_{k-1}, B_{i}$ an $m_{i} \times m_{i-1}$ matrix with entries in $a(p)$, we write $0 \in\left\langle B_{1}, \ldots, B_{k-1}\right\rangle$ if $B_{1}, \ldots, B_{k-1}$ admit a geometric realization. A necessary condition for $0 \in\left\langle B_{1}, \ldots, B_{k-1}\right\rangle$ is that $B_{i+1} B_{i}=0$. If $k=3$ this condition is sufficient.

Let $0 \in\left\langle B_{1}, \ldots, B_{k}\right\rangle$. A stable $k$-order cohomology operation $\varphi$ associated with $B_{1}, \ldots, B_{k}$ is an operation on $m_{0}=l\left(I_{0}\right)$ variables with $m_{k}=$ $l\left(I_{k}\right)$ values given by the universal example (in the sense of [1]) $\left\langle x, E_{k}, y\right\rangle$, where $E_{k}$ is obtained from a $k+1$ stage Postnikov system ( $\left.\mathscr{G}\right)$ realizing $B_{1}, \ldots, B_{k}$ geometrically, and where 


$$
x=r_{k-1}{ }^{*} r_{k-2}{ }^{*} \ldots r_{1}^{*} \iota_{0}, \quad y=h_{k}^{*} \iota_{k} .
$$

If $X$ is a $C W$ complex, the domain $D(\varphi)$ of $\varphi$ consists of all vectors $z$ of length $l\left(I_{0}\right)$ of cohomology classes in $H^{*}\left(X, Z_{p}\right)$ with the property that

$$
f_{z}^{(1)}: X \rightarrow E_{1}, \quad f_{z}^{(1) *} \iota_{0}=z,
$$

can be lifted to $f_{z}^{(k)}: X \rightarrow E_{k}$. The operation $\varphi(z)$ is then the set $\left\{f_{z}^{(k) *}(y)\right\}, f_{z}^{(k)}$ running over all such liftings.

A similar situation occurs when $E_{1}$ is not $K\left(Z_{p}, I\right)$ but $K\left(Z_{p^{r}}, I\right)$. The operation $\varphi$ is then referred to as being a $Z_{p^{r}}-Z_{p}$ operation as $D(\varphi) \subset H^{*}\left(\cdot, \mathbf{Z}_{p^{r}}\right)$ while $\varphi \subset H^{*}\left(\cdot, \mathbf{Z}_{p}\right)$. In this study, we restrict ourselves to $Z_{4}-Z_{2}$ operations defined on a single class (that is, $l\left(I_{0}\right)=1$ ) with a single value $\left(l\left(I_{k}\right)=1\right)$ and $k \leqq 3$. One only has to note that in this case, the condition $B_{2} B_{1}=0$ should read $B_{2} B_{1} \equiv 0 \bmod \left(a(2) S q^{1}\right)$.

Throughout this paper we shall consider only $Z_{2}$ Postnikov approximations, that is: we consider only the $Z_{2^{m}}-k$ invariants or the integral $k$-invariants of order $2^{m}$.

\section{Some relations among high-order operations.}

We consider here two high-order $Z_{4}-Z_{2}$ operations: A third-order operation $\varphi$ and a secondary operation $\bar{\varphi}$.

Let

$$
\begin{aligned}
& B_{1}=\left(\begin{array}{l}
S q^{2} \\
S q^{6}
\end{array}\right), \\
& B_{2}=\left(\begin{array}{ll}
S q^{2} & 0 \\
0 & S q^{2} \\
S q^{7}+S q^{4,2,1} & S q^{2,1}
\end{array}\right), \\
& B_{3}=\left(\begin{array}{lll}
S q^{4,2} & S q^{2} & S q^{1}
\end{array}\right) .
\end{aligned}
$$

2.1 Lemma. $0 \in\left\langle B_{1}, B_{2}, B_{3}\right\rangle$.

Proof. In order to prove this lemma one should construct a threestage Postnikov system:

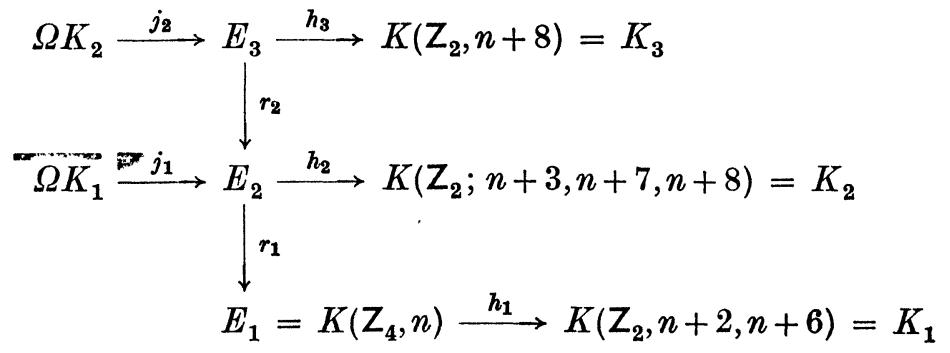




$$
h_{1}^{*}=B_{1} \varrho_{2},
$$

(where $\varrho_{2}: H^{*}\left(\cdot, \mathrm{Z}_{4}\right) \rightarrow H^{*}\left(\cdot, \mathrm{Z}_{2}\right)$ is the reduction),

$$
j_{i-1}^{*} h_{i}^{*}=B_{i}, \quad i=2,3 .
$$

Instead, one seeks a $Z_{2}-Z_{2}$ geometric realization for $\tilde{B}_{1}, \tilde{B}_{2}$, and $\tilde{B}_{3}$, where

$$
\begin{gathered}
\tilde{B}_{1}=\left(\begin{array}{c}
S q^{1} \\
S q^{2} \\
S q^{6}
\end{array}\right), \\
\tilde{B}_{2}=\left(\begin{array}{ccc}
S q^{3} & S q^{2} & 0 \\
S q^{7}+S q^{4,2,1} & 0 & S q^{2} \\
S q^{6,2} & S q^{7}+S q^{4,2,1} & S q^{2,1}
\end{array}\right), \\
\tilde{B}_{3}=B_{3}=\left(S q^{\mathbf{4}, 2} S q^{2} S q^{1}\right) .
\end{gathered}
$$

That is, one seeks a three-stage Postnikov system:

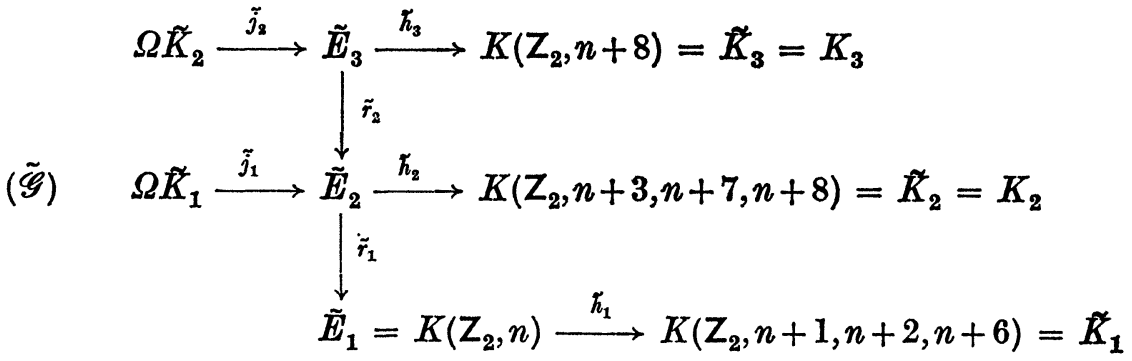

$$
\begin{aligned}
& \tilde{h}_{1}^{*}=\tilde{B}_{1} \quad \text { and } \quad \tilde{j}_{i-1} * \tilde{h}_{i}^{*}=\tilde{B}_{i}, \quad i=2,3 .
\end{aligned}
$$

Once such a realization is established one gets the following comparison:

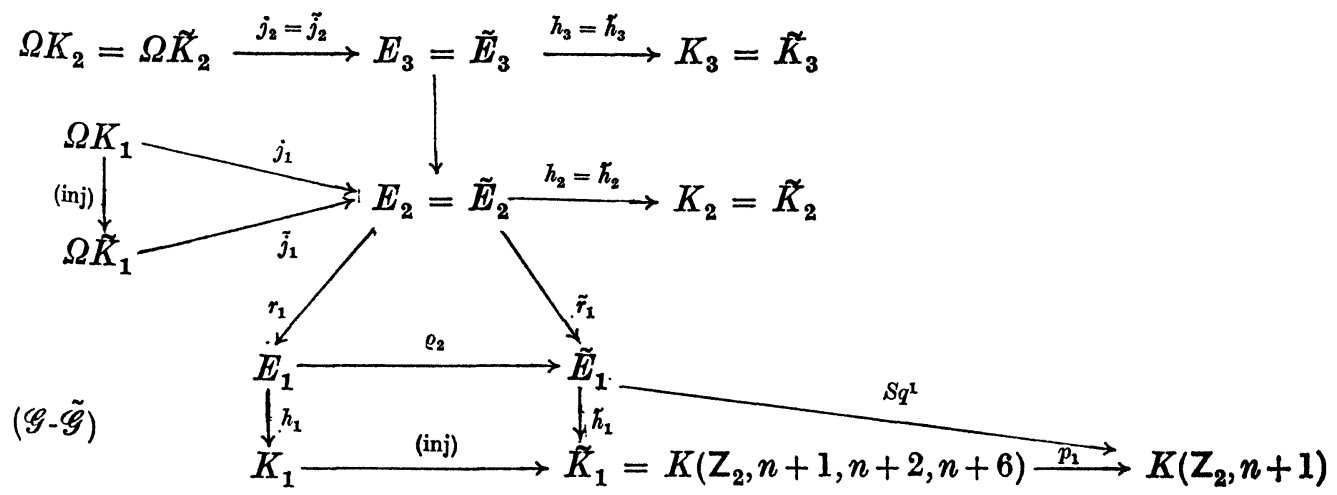


where $p_{1}$ is the projection and $E_{i}, K_{i}, r_{i}, h_{i}, j_{i}$ are the desired realization as

$$
(\mathrm{inj})^{*}=\left(\begin{array}{ll}
0 & 0 \\
1 & 0 \\
0 & 1
\end{array}\right) .
$$

To show $0 \in\left\langle\tilde{B}_{1}, \tilde{B}_{2}, \tilde{B}_{3}\right\rangle$ (or equivalently the existence of $(\tilde{\mathscr{G}})$ ) one realizes that $\tilde{B}_{2} \tilde{B}_{1}=0$. Hence $0 \in\left\langle\tilde{B}_{1}, \tilde{B}_{2}\right\rangle$ and there exists a partial realization

( $\tilde{\mathscr{G}}$-partial)

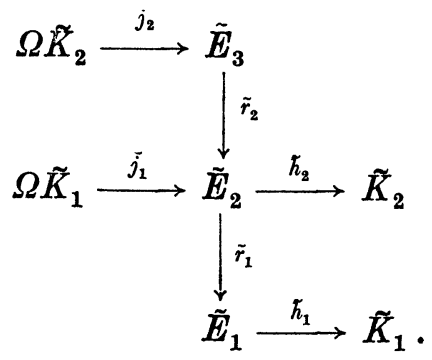

As $\tilde{B}_{3} \tilde{B}_{2}=0$ if $\sigma^{*} \tilde{\iota}_{1}$ and $\tilde{\iota}_{2}$ are the fundamental vectors of $H^{*}\left(\Omega \tilde{K}_{1}, Z_{2}\right)$ and $H^{*}\left(\tilde{K}_{2}, Z_{2}\right)$ respectively,

$$
\tilde{j}_{1} * \tilde{h}_{2} * \tilde{B}_{3} \tilde{\iota}_{2}=\tilde{B}_{3} \tilde{B}_{2} \sigma^{*} \tilde{\iota}_{1}=0 .
$$

Hence $\tilde{h}_{2} * \tilde{B}_{3} \tilde{\imath}_{2} \in \operatorname{ker} \tilde{j}_{1}^{*}$, therefore,

$$
\tilde{h}_{2} * \tilde{B}_{3} \tilde{\iota}_{2} \in \tilde{r}_{1} * P H^{(n+9)}\left(\tilde{E}_{1}, Z_{2}\right) \text { and } \tilde{h}_{2} * \tilde{B}_{3} \tilde{\iota}_{3}=\tilde{r}_{1} * \alpha \tilde{\iota}_{1} \text {, }
$$

$\alpha \in a(2), \operatorname{deg} \alpha=9$. Now, as

$$
\begin{gathered}
S q^{9}=\left(S q^{8}+S q^{6,2}\right) S q^{1}+\left(S q^{7}+S q^{4,2,1}\right) S q^{2}, \quad S q^{8,1}=S q^{8} S q^{1} \\
S q^{7,2}=S q^{7} S q^{2}, \quad S q^{6,3}=S q^{6,1} S q^{2}, \quad S q^{6,2,1}=S q^{6,2} S q^{1}
\end{gathered}
$$

form a basis for $a(2)$ in $\operatorname{dim} 9$,

$$
\alpha=\alpha_{1} S q^{1}+\alpha_{2} S q^{2} \quad \text { and } \quad \alpha \tilde{l}_{1} \in \operatorname{ker} \tilde{r}_{1}^{*} .
$$

It follows that

$$
\tilde{h}_{2} * \tilde{B}_{3} \tilde{\iota}_{2}=0, \quad \tilde{B}_{3} \tilde{\iota}_{2} \in \operatorname{ker} \tilde{h}_{2}^{*}
$$

and consequently

$$
\tilde{B}_{3} \sigma^{*} \tilde{\imath}_{2} \in \operatorname{im} \tilde{j}_{2}^{*}
$$

where $\sigma^{*} \tilde{\imath}_{2}$ is the fundamental vector in $H^{*}\left(\Omega \tilde{K}_{2}, Z_{2}\right)$, and $(\tilde{\mathscr{G}}$-partial) can be completed to $(\tilde{\mathscr{G}})$ by adding $\tilde{h}_{3}: \tilde{E}_{3} \rightarrow \tilde{K}_{3}, \tilde{j}_{2} * \tilde{h}_{3} * \tilde{\imath}_{3}=\tilde{B}_{3} \sigma^{*} \iota_{2}$.

2.1.1. Remark. Note that during the proof of 2.1 the arbitrary original choice of $h_{2}{ }^{*} \iota_{2}$ satisfying 


$$
\tilde{j}_{1} * \tilde{h}_{2} * \iota_{2}=\tilde{j}_{1} * h_{2} * \iota_{2}=\tilde{B}_{2} \sigma^{*} \iota_{1}
$$

was not altered and hence $h_{2}{ }^{*} \iota_{2}$ can be freely changed by any element in $\operatorname{im} \tilde{r}_{1}{ }^{*}$.

Let

$$
\bar{B}_{1}=\left(\begin{array}{c}
S q^{2} \\
S q^{4}
\end{array}\right), \quad \bar{B}_{2}=\left(S q^{2,1}, S q^{1}\right) .
$$

As $\bar{B}_{2} \bar{B}_{1} \equiv 0 \bmod a(2) S q^{1}$, one gets a geometric realization of $0 \in\left\langle\bar{B}_{1}, \bar{B}_{2}\right\rangle$ as follows:

$$
\begin{aligned}
& \begin{aligned}
\left(\overline{\mathscr{G}}_{1}\right) \quad & \left.\right|_{\bar{K}_{1}} \stackrel{\bar{j}_{1}}{\longrightarrow} \bar{E}_{2} \stackrel{\bar{h}_{2}}{\longrightarrow} \bar{K}_{2}=K\left(\mathrm{Z}_{2}, n+4\right) \\
& \bar{E}_{1}=K\left(\mathrm{Z}_{4}, n\right) \stackrel{\bar{h}_{1}}{\longrightarrow} \bar{K}_{1}=K\left(\mathrm{Z}_{2}, n+2, n+4\right) .
\end{aligned} \\
& \bar{h}_{1}^{*}=\bar{B}_{1}, \quad \bar{\jmath}_{1} * \bar{h}_{2} *=\bar{B}_{2} .
\end{aligned}
$$

Our main concern in this section is to choose $h_{2}$ and $h_{3}$ in $(\mathscr{G})$ in such a way that the third-order operation $\varphi$ defined by $(\mathscr{G})$ and the secondary operation $\bar{\varphi}$ defined by $(\overline{\mathscr{G}})$ will satisfy the conditions described in the following propositions 2.2 and 2.3.

Let $B S p^{(k)}$ be the Postnikov approximation of $B S p$ in $\operatorname{dim} \leqq k$ :

$$
\pi_{m}(B S p) \stackrel{\approx}{\longrightarrow} \pi_{m}\left(B S p^{(k)}\right) \text { for } m \leqq k
$$

and $\pi_{m}\left(B S p^{(k)}\right)=0$ for $m>k$. Denote $B=B S p^{(14)}$, let $j: B \rightarrow B S p^{(13)}$ and let $\theta: \widehat{B} \rightarrow B$ be the $K(Z, 3)$ principal fibration induced by $\tilde{g}_{1}: B \rightarrow$ $K(Z, 4)$, where $\tilde{g}_{1}{ }^{*} \iota_{4}$ is a generator.

2.2. Proposition. For every choice of a non-decomposable generator $z$ in $H^{8}\left(B S p^{(13)}, Z_{4}\right), h_{2}$ and $h_{3}$ in $(\mathscr{G})$ can be so chosen that one gets a commutative diagram

$\left(\mathscr{G}_{1}\right)$

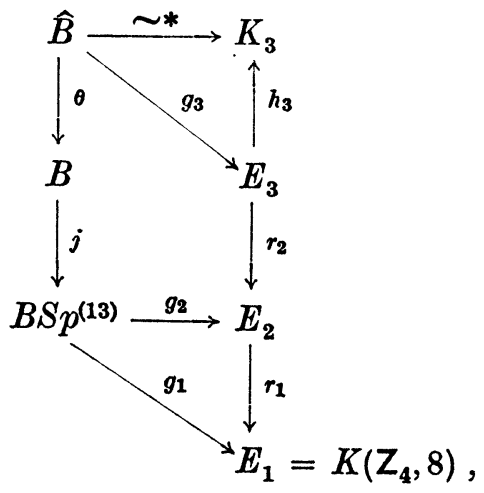

with $g_{1}{ }^{*} \iota_{1}=z$, and $E_{i}, h_{i}$ and $r_{i}$ from $(\mathscr{G})$ for $n=8$. 
2.3. Proposition. With respect to the choices of $h_{i}$ in $(\mathscr{G})$ made in 2.2, $\bar{h}_{2}$ in $(\bar{G})$ can be chosen so that the following commutative diagram is obtained:

$\left(\mathscr{G}_{2}\right)$

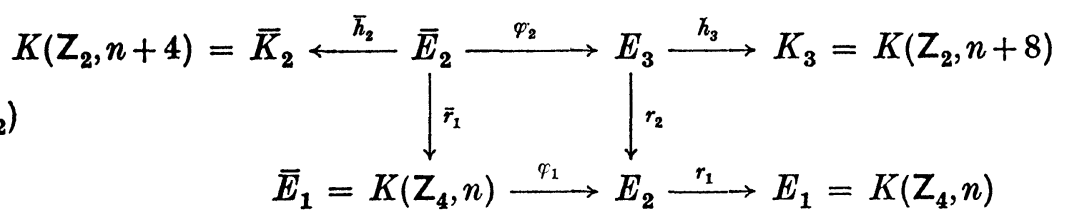

with $\varphi_{1}{ }^{*} r_{1}{ }^{*} \iota_{1}=2 \bar{\iota}_{1}$ and $\varphi_{2}{ }^{*} h_{3} * \iota_{3}=S q^{4} \bar{h}_{2} * \bar{\iota}_{2}+S q^{8} \varrho_{2} \bar{r}_{1}^{*} \bar{\iota}_{1}$, where $\iota_{1}, \bar{\iota}_{1}, \bar{\iota}_{2}$, and $\iota_{3}$ are the fundamental vectors.

Proof of 2.2. One can obviously obtain the following part of $\left(\mathscr{G}_{1}\right)$ :

$\left(\mathscr{G}_{1}\right)$ part
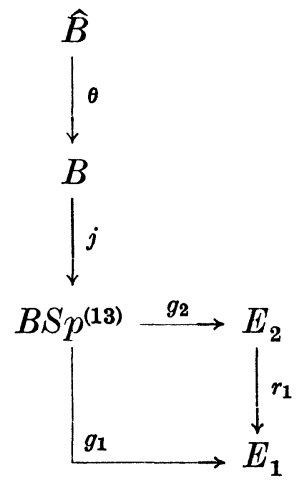

Note that $H^{k}\left(B S p, \mathrm{Z}_{2}\right) \approx H^{k}\left(B S p^{(13)}, \mathrm{Z}_{2}\right)$ for $k \leqq 14$ and hence $S q^{2} z=0$, $S q^{6} z=0$.

If $\mu_{B}$ and $\Delta_{B}$ denote the multiplication $B \times B \rightarrow B$ and diagonal $B \rightarrow B \times B$, put $\lambda_{2}=\mu_{B} \Delta_{B}$. As $\pi_{15}(B S p)=0$ and as the $k$-invariant of $B S p$ in $\operatorname{dim} 17$ is integral of order divisible by 4 ,

$$
H^{*}\left(B, \mathrm{Z}_{4}\right)=H^{*}\left(B S p^{(15)}, \mathrm{Z}_{4}\right)=H^{*}\left(B S p, \mathrm{Z}_{4}\right)=\mathbf{Z}_{4}\left[w_{4}, w_{8}, w_{12}, w_{16}\right]
$$

in $\operatorname{dim} \leqq 16$ and

it follows that

$$
\mu_{B}^{*} w_{4 k}=\sum_{j=0}^{k} w_{4 j} \otimes w_{4(k-j)}, \quad w_{0}=1
$$

$$
\begin{gathered}
\lambda_{2} * w_{4}=2 w_{4}, \quad \lambda_{2} * w_{8}=2 w_{8}+w_{4}^{2}, \\
\lambda_{2} * w_{12}=2 w_{12}+2 w_{8} w_{4}, \\
\lambda_{2} * w_{16}=2 w_{16}+2 w_{4} w_{12}+w_{8}{ }^{2} .
\end{gathered}
$$

If $j^{*} z= \pm w_{8}+k w_{4}^{2}, k \in Z_{4}$, then $\lambda_{2}{ }^{*} j^{*} z=2 w_{8} \pm w_{4}^{2}$ is primitive. Consequently $g_{1} \circ j \circ \lambda_{2}$ is an $H$-mapping, and as $H^{k}\left(B \wedge B, Z_{2}\right)=0$ for $k=9,13$, 
it follows that $\left[B \wedge B, \Omega K_{1}\right]=0$ and $g_{2} \circ j \circ \lambda_{2}$ is an $H$-mapping too. As $P H^{k}\left(B, \mathrm{Z}_{2}\right)=0, k=11,15$, and $P H^{16}\left(B, \mathrm{Z}_{2}\right)$ is generated by $\varrho_{2} w_{4}^{4}$, it follows that

$$
\left(g_{2} \circ j \circ \lambda_{2}\right) * h_{2} * \iota_{2}=\left(0,0, \varepsilon \varrho_{2} w_{4}^{4}\right), \quad \varepsilon \in Z_{2} .
$$

Changing $h_{2}$ in $(\mathscr{G})$ if necessary so that $h_{2}{ }^{*} \iota_{2}$ is altered by $S q^{8} \tilde{r}_{1} * \tilde{\imath}_{1}=$ $S q^{8} \varrho_{2} r_{1}{ }^{*} \iota_{1}$ (and see remark 2.1.1) one may assume that $\left(g_{2} \circ j \circ \lambda_{2}\right) * h_{2}{ }^{*} \iota_{2}=0$.

Let $r_{2}{ }^{\prime}: E_{3}{ }^{\prime} \rightarrow E_{2}$ be the fibration induced by

$$
p_{12} \circ h_{2}: E_{2} \rightarrow K\left(Z_{2}, n+3, n+7\right),
$$

where $p_{12}: K\left(Z_{2} ; n+3, n+7, n+8\right)=K_{2} \rightarrow K\left(Z_{2}, n+3, n+7\right)$ is the projection. Now, if $h_{2}{ }^{*} \iota_{2}=\left(v_{1}, v_{2}, v_{3}\right)$, then $\left(p_{12} \circ h_{2}\right)^{*}=\left(v_{1}, v_{2}\right)$, and hence $v_{1}, v_{2} \in \operatorname{ker} r_{2}{ }^{*}$. Further, as

$$
0=B_{3} h_{2}^{*} \iota_{2}=S q^{4,2} v_{1}+S q^{2} v_{2}+S q^{1} v_{3},
$$

$S q^{1} v_{3} \in \operatorname{ker} r_{2}{ }^{*}$. It follows that $r_{2}{ }^{*} h_{2}{ }^{*} \iota_{2}=\left(0,0, \varrho_{2} v\right)$ for some class $v \in H^{n+8}\left(E_{3}{ }^{\prime}, \mathbf{Z}_{4}\right)$.

One gets the following diagrams:

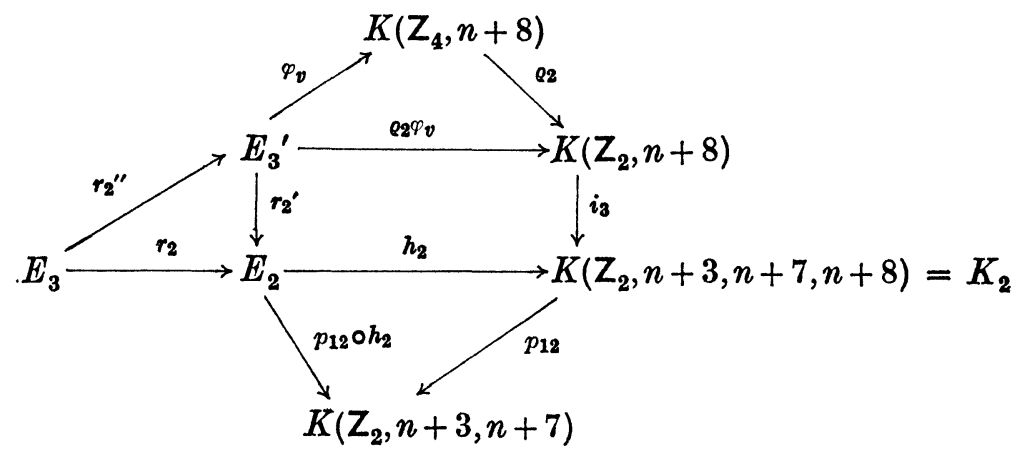

where $\varphi_{v}{ }^{*} \iota_{n+8}=v$ and $r_{2}{ }^{\prime \prime}$ is the fiber of $\varrho_{2} \varphi_{v}$,

$\left(\mathscr{G}_{3}\right)$

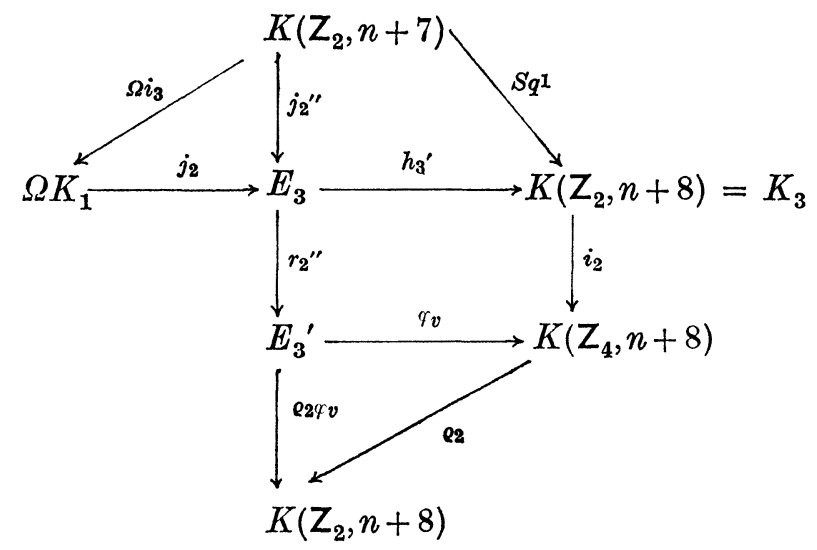


As $h_{3} j_{2}{ }^{\prime \prime} \sim h_{3} \circ j_{2} \circ \Omega i_{3} \sim h_{3}{ }^{\prime} j_{2}{ }^{\prime \prime}$ if $\iota_{3} \in H^{*}\left(K_{3}, Z_{2}\right)$ is the fundamental class, then

$$
\left(h_{3}{ }^{*}-h_{3}{ }^{*}\right) \iota_{3} \in \operatorname{ker} j_{2}{ }^{\prime *} *, \quad\left(h_{3}{ }^{*}-h_{3}{ }^{*}\right) \iota_{3}=r_{2}{ }^{\prime *} v_{1}{ }^{\prime}
$$

where $v_{1}{ }^{\prime} \in H^{n+8}\left(E_{3}{ }^{\prime}, \mathrm{Z}_{2}\right)$. Altering $\varphi_{v}$ (and hence $v$ ) by $i_{2} \varphi_{v_{1}}$, where $\varphi_{v_{1}{ }^{\prime}}: E_{3}{ }^{\prime} \rightarrow K_{3}$ and $\varphi_{v_{1}}^{*} \iota_{3}=v_{1}{ }^{\prime}, \varrho_{2} v$ is not altered and one may assume $h_{3}{ }^{\prime}=h_{3}$. Moreover, any further alteration of $v$ by an element in $r_{2}{ }^{\prime *}\left(\operatorname{ker} \varrho_{2}\right)$ will correspond to a change of $h_{3}=h_{3}{ }^{\prime}$ in $(\mathscr{G})$ without changing the relation $j_{2} * h_{3} *=B_{3}$.

Return now to the case $n=8$ and diagram $\left(\mathscr{G}_{1}\right)_{\text {part }}$. As $H^{k}\left(B, Z_{2}\right)=0$, $k=11,15$, it follows that $g_{2} \circ j$ lifts to

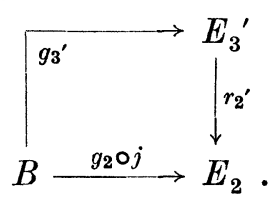

As $\left[B \wedge B, K\left(Z_{2}, 10,14\right)\right]=0, g_{3}{ }^{\prime} \circ \lambda_{2}$ is an $H$-mapping. As $v$ is primitive, $\lambda_{2}{ }^{*} g_{3}{ }^{*} v=2 a w_{4}{ }^{4}\left(a=0\right.$ or 1 in $\left.\mathrm{Z}_{4}\right)$.

If $\iota_{0} \in H^{n}\left(E_{1}, \mathrm{Z}_{4}\right)=H^{n}\left(K\left(\mathrm{Z}_{4}, n\right), \mathrm{Z}_{4}\right)$ is the fundamental class $S q^{9} \varrho_{2} r_{1}{ }^{*} \iota_{0}$ $=0$ for all $n$, then

$$
S q^{8} \varrho_{2} r_{1}^{*} \iota_{0}=\varrho_{2} \bar{v} \quad \text { for some } \quad \bar{v} \in H^{n+8}\left(E_{2}, \mathrm{Z}_{4}\right),
$$

and for $n=8$,

$$
S q^{8} \varrho_{2} r_{1}{ }^{*} \iota_{0}=\varrho_{2}\left(r_{1}^{*} \iota_{0}\right)^{2}=\varrho_{2} \bar{v} .
$$

It follows that $2\left(r_{1} \iota_{0}\right)^{2}=2 \bar{v}$. Now,

and

$$
\lambda_{2}{ }^{*} g_{3}{ }^{*} r_{2}{ }^{*}\left(r_{1}{ }^{*} \iota_{0}\right)^{2}=\lambda_{2}{ }^{*} j^{*} g_{1}{ }^{*} \iota_{0}{ }^{2}=\lambda_{2}{ }^{*}\left( \pm w_{8}+k w_{4}{ }^{2}\right)^{2}=w_{4}{ }^{4}
$$

$$
\lambda_{2}{ }^{*} g_{3}{ }^{*}{ }_{2}{ }^{*}(2 a \bar{v})=\lambda_{2}{ }^{*} g_{3}{ }^{*} r_{2}{ }^{*} 2 a\left(r_{1}{ }^{*} \iota_{0}\right)^{2}=2 a w_{4}{ }^{4} .
$$

Replacing $v$ by $v+2 a r_{2}{ }^{*} \bar{v}$ if necessary, we may assume

$$
\lambda_{2}{ }^{*} g_{3}{ }^{*} v=0 \text {. }
$$

As $g_{3}{ }^{*} v \in \operatorname{ker} \lambda_{2}{ }^{*}, \operatorname{dim} v=16$, it follows that $g_{3}{ }^{*} v$ must be in the ideal generated by $w_{4}$ and hence, $g_{3}{ }^{*} v \in \operatorname{ker} \theta^{*}$. Using this fact, diagrams $\left(\mathscr{G}_{3}\right)$ and $\left(\mathscr{G}_{4}\right)$ for $n=8$ yield

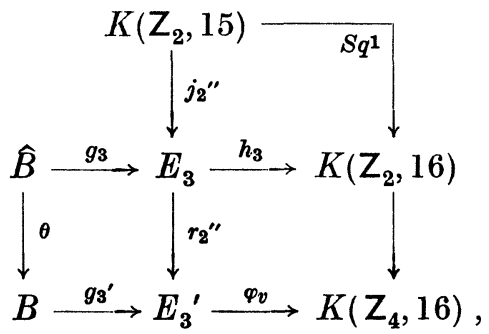


with $\varphi_{v} \circ g_{3}{ }^{\circ} \circ \theta \sim *$. As $h_{3} \circ g_{3}$ can be lifted to $D: \widehat{B} \rightarrow K\left(Z_{2}, 15\right)$, a change in $g_{3}$ by $j_{2}^{\prime \prime} \circ D$ yields $h_{3} \circ g_{3} \sim *$, and 2.2 follows.

As a side result of this proof one gets (a) and therefore one has

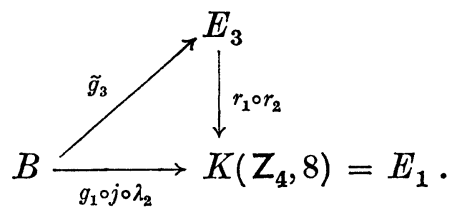

As it was done for $g_{3}$, the map $\tilde{g}_{3}$ can be chosen so that $h_{3} \circ \tilde{g}_{3} \sim *$. If $i: B S^{3} \rightarrow B$ is induced by

$$
B S^{3}=B S p(1) \subset B S p \rightarrow B
$$

one gets $i^{*} \lambda_{2} * \lambda_{2} * \tilde{g}_{3} * h_{3} * \iota_{3}=0$. As

$$
i^{*} \lambda_{2} * \lambda_{2} * j^{*} g_{1}^{*} \iota_{8}=2 w^{2}
$$

where $w \in H^{4}\left(B S^{3}, \mathrm{Z}_{4}\right)$ is a generator, one has:

2.4. Corollary. If $\varphi$ is the third order operation induced by (G) (with the 2.2 choices of $\left.h_{i}\right)$, then $0=\varphi\left(2 w^{2}\right)$ (with 0 indeterminancy).

Proof of 2.3. First consider the following commutative ladder:

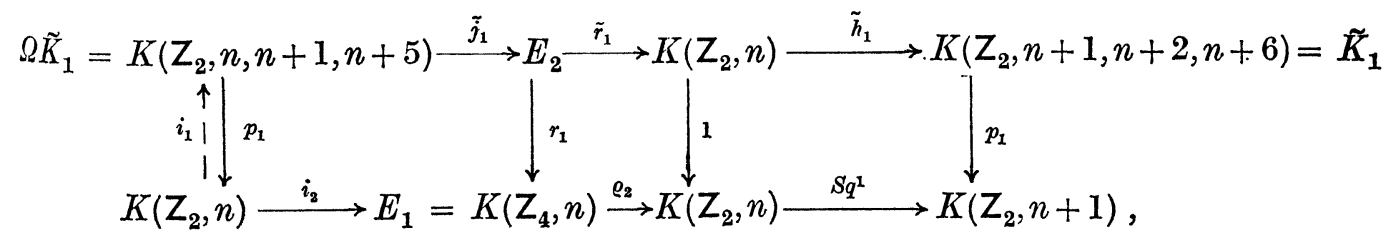

where $\varrho_{2}, \tilde{h}_{1}, E_{2}=\tilde{E}_{2}, \tilde{r}_{1}$ and $\tilde{j}_{1}$ are the same as in diagram $(\tilde{\mathscr{G}})$ of 2.1 . Further $\tilde{j}_{1} * h_{2} * \iota_{2}=\tilde{B}_{2} \tilde{\imath}_{2}$ with $\tilde{B}_{2}$ as in the proof of 2.1. If $i_{1}: K\left(Z_{2}, n\right) \rightarrow$ $\Omega \tilde{K}_{1}$ is the injection, then $\tilde{\varphi}_{1}=\tilde{j}_{1} \circ i_{1}$ is a lifting of $i_{2}$, and

$$
\tilde{\varphi}_{1} * h_{2}{ }^{*} \iota_{2}=i_{1} * \tilde{j}_{1} * h_{2}{ }^{*} \iota_{2}=i_{1} * \tilde{B}_{2} \iota_{2}=\left(\begin{array}{l}
S q^{3} \\
S q^{7}+S q^{4,2,1} \\
S q^{6,2}
\end{array}\right) \tilde{\iota}_{0} .
$$

Put $\varphi_{1}=\tilde{\varphi}_{1} \circ \varrho_{2}$ and note that

$$
\varphi_{1}^{*} r_{1}^{*} \iota_{1}=\varrho_{2} * \tilde{\varphi}_{1}^{*} r_{1}^{*} \iota_{1}=\varrho_{2}^{*} i_{2}^{*} \iota_{1}=2 \bar{\iota}_{1} .
$$


Now consider the following commutative diagram:

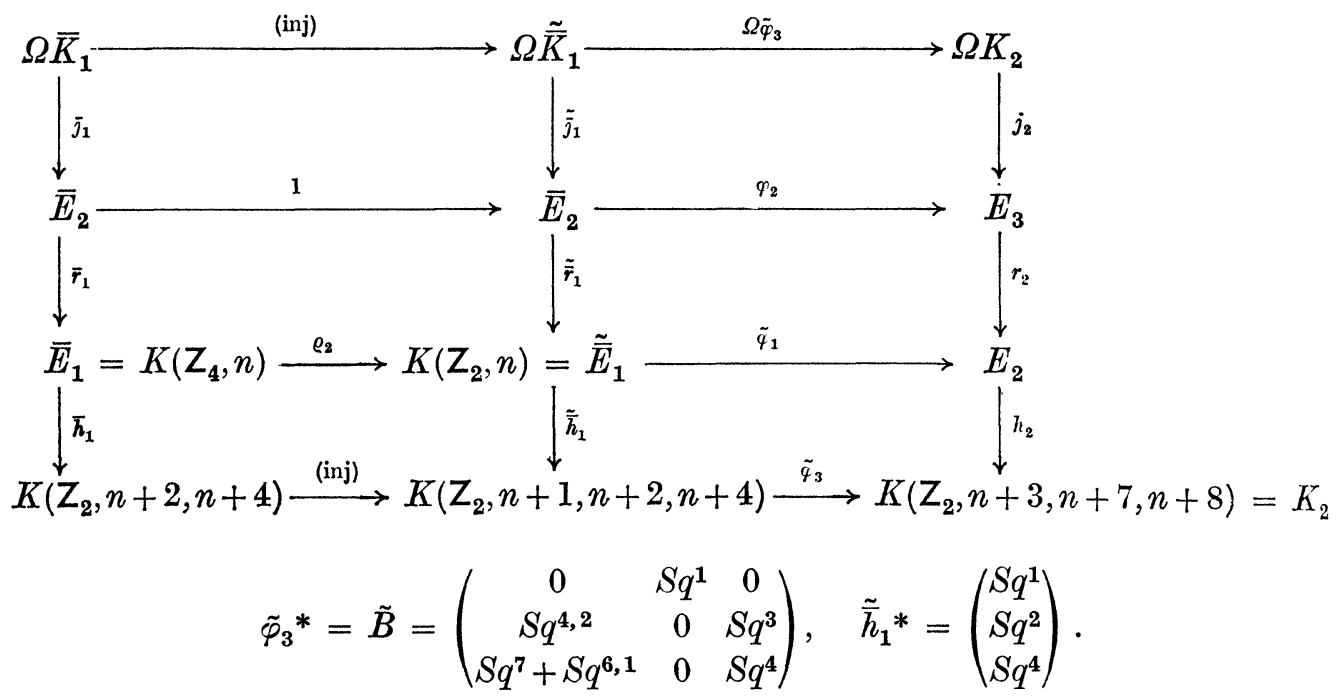

Put $\tilde{\varphi}_{3} \circ($ inj $)=\varphi_{3}$. There exists

$$
z \in H^{n+4}\left(\bar{E}_{2}, \mathrm{Z}_{2}\right), \quad \tilde{\bar{\jmath}}_{1}^{*} z=\left(S q^{4}, S q^{2,1}, S q^{1}\right) \sigma^{*} \tilde{\bar{\iota}}_{1},
$$

and one can choose $\bar{h}_{2}$ so that $z=\bar{h}_{2}{ }^{*} \bar{\iota}_{2}$ and hence

since

$$
\tilde{\bar{\jmath}}_{1} * \bar{h}_{2} *=\left(S q^{4}, S q^{2,1}, S q^{1}\right),
$$

$$
\begin{aligned}
\bar{\jmath}_{1} * \varphi_{2}{ }^{*} h_{3}{ }^{*}=\left(\Omega \varphi_{3}\right) * j_{2} * h_{3} * \iota_{3} & =(\mathrm{inj}) * B_{3} \tilde{B} \sigma^{*} \iota_{1} \\
& =S q^{4}\left(S q^{2,1} S q^{1}\right) \sigma^{*} \bar{\iota}_{1}=S q^{4} \bar{\jmath}_{1}{ }^{*} h_{2} * \bar{\iota}_{2} .
\end{aligned}
$$

Hence it follows that

$$
\varphi_{2}{ }^{*} h_{3}{ }^{*} \iota_{3}+S q^{4} h_{2}^{*} \iota_{2} \in \bar{r}_{1}^{*} P H^{n+8}\left(\bar{E}_{1}, \mathrm{Z}_{2}\right)
$$

and, consequently,

$$
\varphi_{2}{ }^{*} h_{3}{ }^{*} \iota_{3}=S q^{4} h_{2}{ }^{*} \bar{\iota}_{2}+\varepsilon S q^{8} \varrho_{2} \bar{r}_{1} * \tilde{\iota}_{0}, \quad \varepsilon \in \mathrm{Z}_{2}, \tilde{i}_{0} \in H *\left(\bar{E}_{1}, \mathrm{Z}_{2}\right) .
$$

If one shows that $\bar{\varphi}\left(w^{2}\right)=\varrho_{2} w^{3}$, where $w \in H^{4}\left(B S^{3}, Z_{4}\right)$ is a generator, then by 2.4 , one obtains

$$
0=\varphi\left(2 w^{2}\right)=S q^{4} \bar{\varphi}\left(w^{2}\right)+\varepsilon S q^{8} \varrho_{2} w_{4}{ }^{2}=(1+\varepsilon) \varrho_{2} w_{4}{ }^{4} .
$$

Hence $\varepsilon=1$, and 2.3 follows.

Now, the relation $\bar{\varphi}\left(w^{2}\right)=\varrho_{2} w^{3}$ follows from general calculations carried out independently by Kristensen [6], Gitler and Milgram [3] and others, but as the adaptation of their calculations to this simple 
case is not simpler than a direct proof, we give here an outline of the proof of the following:

2.5. Lemma. Let $\langle x, Y, u\rangle$ be the universal example for the (unique) $\mathrm{Z}-\mathrm{Z}_{2}$ secondary operation induced by the (non-stable) relation

$$
e\left[\left(S q^{2,1} S q^{2}\right) \circ \varrho_{2}\right]>4 \text {. }
$$

Then $\bar{\varrho}_{4} x^{2} \in D(\bar{\varphi})$ and $\bar{\varrho}_{2} x^{3} \in \bar{\varphi}\left(x^{2}\right)$, where $\varrho_{i}: H^{*}(, \mathrm{Z}) \rightarrow H^{*}\left(, \mathrm{Z}_{i}\right)$ is the reduction.

Proof. One has the (once deloopable) commutative diagram:

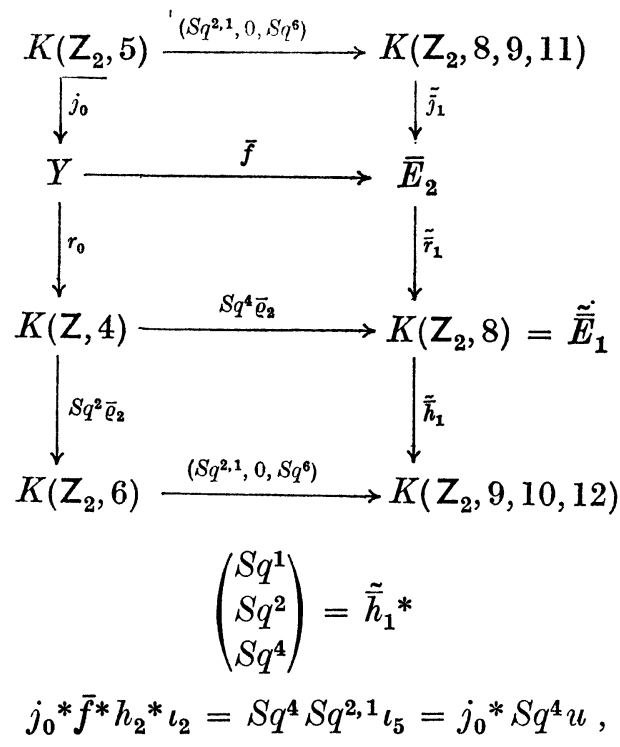

where $j_{0} * u=S q^{2,1} \iota_{5}$. Now, if $\mu_{Y}$ is the multiplication in $Y$, one has

$$
\mu_{Y^{*}} u=u \otimes 1+1 \otimes u+\bar{\varrho}_{2} x \otimes \bar{\varrho}_{2} x, \quad x=r_{0}{ }^{*} \iota_{4} .
$$

It follows that

$S q^{4} u+\bar{\varrho}_{2} x^{3}+\bar{f}^{*} h_{2}{ }^{*} \iota_{2} \in P H^{12}\left(Y, \mathrm{Z}_{2}\right) \cap \operatorname{ker} j_{0}{ }^{*}=r_{0}{ }^{*} P H^{12}\left(K(\mathrm{Z}, 4), \mathrm{Z}_{2}\right)=0$.

Changing $\bar{f}$ so that $\bar{f}^{*} h_{2}^{*}$ is altered by $S q^{4} u$, that is, altering $\bar{f}$ by $\alpha: Y \rightarrow K\left(Z_{2}, 8,9,11\right), \alpha^{*} \iota_{8}=u, \alpha^{*} \iota_{j}=0, j=9,11$, one gets $\bar{\varrho}_{2} x^{3} \in \bar{\varphi}\left(x^{2}\right)$.

\section{The proof of the main theorem.}

Let $X, \mu$ be an $H$-space satisfying (1) and (2).

Suppose $P H^{14}\left(X, Z_{2}\right)=0$. Let $\hat{\theta}: \hat{X} \rightarrow X$ be the $K(Z, 2)$-principal fibra- 
tion induced by $\hat{g}_{0}: X \rightarrow K(\mathrm{Z}, 3), \hat{g}_{0}$ inducing isomorphism of $H^{3}(\cdot, \mathrm{Z})$ torsion.

3.1 Lemma. $H^{*}\left(\hat{X}, \mathrm{Z}_{2}\right)=\left[H^{*}\left(X, \mathrm{Z}_{2}\right) /\left(x_{3}\right)\right] \otimes \mathrm{Z}_{2}\left(\widehat{x}_{4}\right) \otimes \Lambda\left(S q^{1} \widehat{x}_{4}\right)$ as a Hopf algebra over the Steenrod algebra.

Proof. If $\iota_{3} \in H^{3}(K(Z, 3), Z)$ is the fundamental class, then ker $\hat{g}_{0}{ }^{*}$ is the $a(2)$ ideal generated by $S q^{2} \bar{\varrho}_{2} \iota_{3}$. Hence, $H^{*}\left(\hat{X}, Z_{2}\right) / / \mathrm{im} \hat{\theta}^{*}$ is generated by the algebraic suspension of

$$
S q^{2} \bar{\varrho}_{2} \iota_{3}, \quad S q^{4,2} \bar{\varrho}_{2} \iota_{3}, \ldots, S q^{2^{n}, 2^{n-1}, \ldots 4,2} \bar{\varrho}_{2} \iota_{3}, \ldots, \quad S q^{3} \bar{\varrho}_{2} \iota_{3} .
$$

Consequently

$$
H^{*}\left(\hat{X}, Z_{2}\right) / / \operatorname{im} \hat{\theta}^{*}=Z_{2}\left(\hat{x}_{4}\right) \otimes \Lambda\left(S q^{1} \widehat{x}_{4}\right),
$$

as $S q^{2} S q^{2} \bar{\varrho}_{2}=0, S q^{2} \hat{x}_{4}=0$ and, hence, $S q^{4,1} \hat{x}_{4}=S q^{5} \widehat{x}_{4}=0$. It follows that

$$
H^{*}\left(\hat{X}, \mathbf{Z}_{2}\right) \approx H^{*}\left(X, \mathrm{Z}_{2}\right) / /\left(x_{3}\right) \otimes \mathrm{Z}_{2}\left[\hat{x}_{4}\right] \otimes \Lambda\left(S q^{1} \hat{x}_{4}\right)
$$

as algebras. Since $\hat{x}_{4}$ is obviously primitive, the above is actually an isomorphism of Hopf algebras. The only possible non-trivial extension over $a(2)$ of the above splitting is given by $S q^{2,1} \widehat{x}_{4}=\varepsilon \hat{\theta} * x_{7}$.

Now, $X$ can be mapped into $S p^{(5)} \approx S p^{(6)}$. Moreover,

$$
X^{(5)} \approx S p^{(5)} \approx\left(S^{3}\right)^{(5)}
$$

where $Y^{(k)}$ is the Postnikov approximation of $Y$. Since

$$
Z_{2} \approx{ }^{2} \pi_{6}(X) \neq{ }^{2} \pi_{6}\left(S p^{(6)}\right)=0,
$$

one has a fibration

$$
X^{(6)} \stackrel{f^{(6)}}{\longrightarrow} S p^{(6)} \stackrel{h^{(6)}}{\longrightarrow} K\left(Z_{2}, 7\right) .
$$

where $h^{(6) *} \iota_{7}$ restricts to $\varrho_{2} \sigma^{*} w_{8} \in H^{7}\left(S p^{(6)}, Z_{2}\right)$. Lifting $f^{(6)}$ to $\hat{f}^{(6)}: \hat{X} \rightarrow$ $\hat{S} p^{(6)}=S p^{(6)}$ made 3-connected, one has

$$
H^{*}\left(\widehat{S p^{(6)}}, \mathrm{Z}_{2}\right)=\mathrm{Z}_{2}\left[h^{(6) *} \iota_{7}\right] \otimes \mathrm{Z}_{2}\left[\widehat{w}_{4}\right] \otimes \Lambda\left(S q^{1} \widehat{w}_{4}\right)
$$

in $\operatorname{dim} \leqq 7$,

$$
S q^{2,1} \hat{w}_{4}=h^{(6) *} \iota_{7} \quad \text { and } \quad \hat{f}^{(6) *} \hat{w}_{4}=\hat{x}_{4} .
$$

Hence $S q^{2,1} \widehat{x}_{4}=0$, and 3.1 follows.

3.2 Corollary. $Q H^{*}\left(X, \mathrm{Z}_{2}\right) / P H^{*}\left(X, \mathrm{Z}_{2}\right) \approx Q H^{*}\left(\hat{X}, \mathrm{Z}_{2}\right) / P H^{*}\left(\hat{X}, \mathrm{Z}_{2}\right)$, where $Q A$ stands for the module of indecomposables. 
3.3. Lemma. Let $B_{2}(X)$ and $B_{2}(\hat{X})$ be the projective planes of $X$ and $\hat{X}$, respectively. There exists a commutative diagram

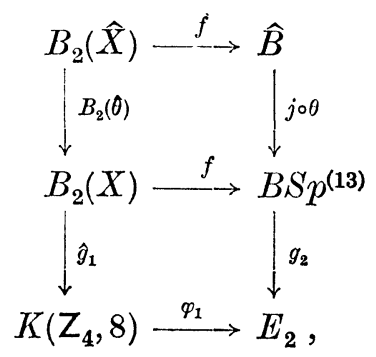

where $g_{2}$ and $\varphi_{1}$ are given in 2.2 and 2.3 , respectively, and

$$
\varrho_{2} \sigma^{*} \hat{g}_{1} * \iota_{0}=x_{7}
$$

where $\iota_{0} \in H^{8}\left(K\left(\mathrm{Z}_{4}, 8\right), \mathrm{Z}_{4}\right)$ is the fundamental class.

Proof. As in the proof of 3.1 one has a fibration

$$
\left[B_{2}(X)\right]^{(7)} \stackrel{f^{(7)}}{\longrightarrow} B S p^{(7)} \rightarrow K\left(Z_{2}, 8\right)
$$

and hence, a diagram

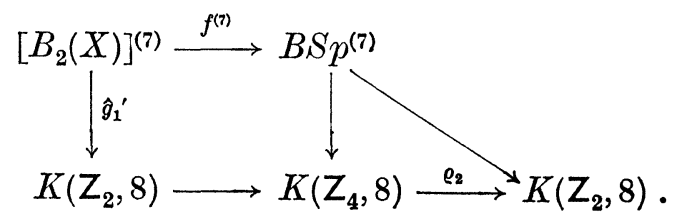

Since $\hat{g}_{1}{ }^{*} \iota_{8}$ is a reduction of a $\mathrm{Z}_{4}$ class in $H^{*}\left(B_{2}(X), \mathrm{Z}_{2}\right)$, one gets

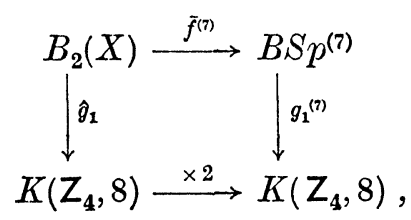

where $g_{1}^{(7)}$ corresponds to $g_{1}$ in 2.2. Now, consider the mappings

$$
B_{1}(X)=\Sigma X \stackrel{i}{\longrightarrow} B_{2}(X) \stackrel{k}{\longrightarrow} B_{2}(X), B_{1}(X)
$$

and the induced sequence of $Z_{2}$ cohomology $H^{*}\left(B_{2}(X), B_{1}(X)\right) \approx \bar{H}^{*}(\Sigma X) \otimes \bar{H}^{*}(\Sigma X) \stackrel{k^{*}}{\longrightarrow} H^{*}\left(B_{2}(X)\right) \stackrel{i}{\longrightarrow} H^{*}\left(B_{1}(X)\right)$. It follows that $H^{*}\left(B_{2}(X), Z_{2}\right)$ is generated (as a $Z_{2}$ module) in $\operatorname{dim}<16$ by 
$u_{4}, u_{8}, u_{4} \cdot u_{8}, k^{*}\left(i^{*} u_{4} \otimes \bar{u}_{11}\right), k^{*}\left(\bar{u}_{11} \otimes i * u_{4}\right) \quad$ and $\quad\left(\Sigma^{*} \bar{u}_{11}=x_{3} x_{7}\right)$.

Note that

$$
\Sigma^{*} i^{*} u_{i+1}=x_{i} \in H^{i}\left(X, Z_{2}\right), i=3,7, \quad \text { and } \hat{g}_{1}{ }^{*} \varrho_{2} \iota_{8}=u_{8} .
$$

All are reductions of integral classes. Hence,

$$
H^{15}\left(B_{2}(X), \mathrm{Z}_{2}\right) \subset \operatorname{ker} B_{2}(\hat{\theta})^{*},
$$

and as $\pi_{11}(B S p)=0$, all $k$-invariants of $B S p$ in dim $\leqq 14$ vanish on $H^{*}\left(B_{2}(X), \pi_{*}(B S p)\right)$. It follows that $f^{(7)}$ can be lifted to $f: B_{2}(X) \rightarrow B S p^{(13)}$ and as

$$
H^{15}\left(B_{2}(X), Z_{2}\right) \subset \operatorname{ker} B_{2}(\hat{\theta})^{*}
$$

one gets $f^{\prime}: B_{2}(\hat{X}) \rightarrow B S p^{(14)}=B$ to obtain the commutative diagram:

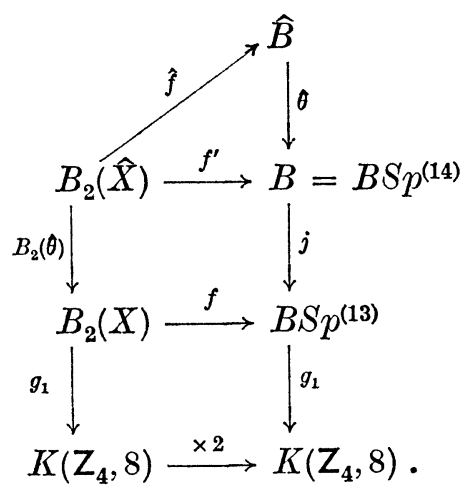

Finally

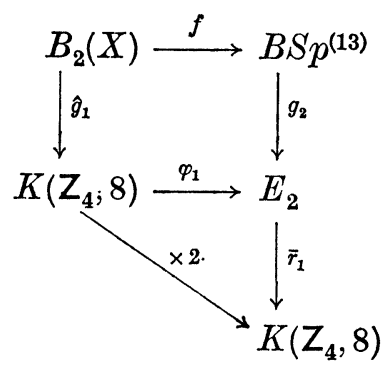

is commutative as

$$
\left[B_{2}(X), \Omega K_{1}\right]=H^{9}\left(B_{2}(X), Z_{2}\right) \oplus H^{13}\left(B_{2}(X), Z_{2}\right)=0,
$$

and 3.3 follows. 
To complete the proof of the main theorem, we consider the diagram

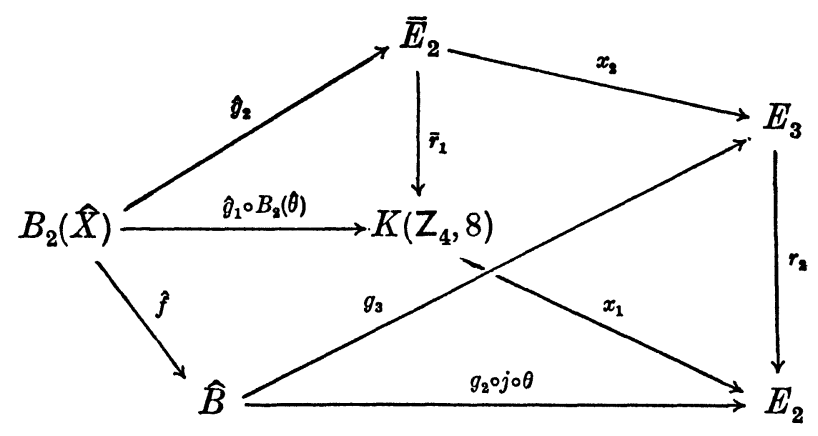

The bottom face of this diagram is commutative by 3.3, the front and right hand faces commutativity follows from 2.2 and 2.3 , respectively, and $g_{3} * h_{3} * \iota_{3}=0$.

As

$$
\hat{g}_{1} * \bar{h}_{1} * \bar{\iota}_{1}=\bar{B}_{1} u_{8}=\left(S q^{2}, S q^{4}\right) u_{8}=\left(0, \varepsilon u_{8} u_{4}\right), \quad \varepsilon \in Z_{2},
$$

$B_{2}(\theta) * \hat{g}_{1} * \bar{h}_{1} * \iota_{1}=0$; and $\hat{g}_{2}$ exists, and the back face of the above diagram is commutative.

Now the difference between $x_{2} \circ \hat{g}_{2}$ and $g_{3} \circ \hat{f}$ can be measured by an element $\alpha$ in

$$
\left[B_{2}(\hat{X}), \Omega K_{2}\right]=H^{10}\left(B_{2}(\hat{X}), \mathrm{Z}_{2}\right) \oplus H^{14}\left(B_{2}(\hat{X}), \mathrm{Z}_{2}\right) \oplus H^{15}\left(B_{2}(\hat{X}), \mathrm{Z}_{2}\right) .
$$

Further, $\hat{k}^{*}: H^{m}\left(B_{2}(\hat{X}), B_{1}(\hat{X}) ; Z_{2}\right) \rightarrow H^{m}\left(B_{2}(\hat{X}), Z_{2}\right)$ is onto for $10 \leqq$ $m<16$, and consequently

$$
\hat{g}_{2}{ }^{*} x_{2}{ }^{*} h_{3}{ }^{*} \iota_{3}+\hat{f}^{*} g_{3}{ }^{*} h_{3}{ }^{*} \iota_{3}=B_{3} \alpha=B_{3} \hat{k}^{*} \bar{\alpha} .
$$

As $g_{3}{ }^{*} h_{3}{ }^{*}=0$ by 2.2 and

$$
x_{2}{ }^{*} h_{3}{ }^{*} \iota_{3}=S q^{4} \bar{h}_{2}{ }^{*} \iota_{2}+S q^{8} \varrho_{2} \bar{r}_{1}^{*} \bar{\iota}_{1}
$$

by 2.3 , and since $S q^{4} H^{12}\left(B_{2}\left(\hat{X}, Z_{2}\right)=0\right.$, one has

$$
B_{3} \hat{k}^{*} \bar{\alpha}=\hat{g}_{2}{ }^{*} S q^{8} \varrho_{2} \bar{r}_{1}^{*} \bar{\imath}_{1}=B_{2}(\hat{\theta}) * u_{8}{ }^{2}=\hat{u}_{8}{ }^{2}
$$

or as $\hat{u}_{8}^{2}=\hat{k} *\left(\hat{\imath} * \hat{u}_{8} \otimes \hat{\imath}^{*}\left(\hat{u}_{8}\right)\right.$, where $\hat{\imath}: B_{2}(\hat{X}) \rightarrow B_{1}(\hat{X})$,

$$
\left.\hat{k}^{*}\left[\hat{\imath}^{*} \hat{u}_{8} \otimes \hat{\imath}^{*} \hat{u}_{8}\right)+B_{3} \bar{\alpha}\right]=0 .
$$

As $\hat{\imath}^{*} \hat{u}_{8} \notin \overline{(i(2)} H^{*}\left(B_{1}(\hat{X}), Z_{2}\right)$,

$$
w=\hat{\imath}^{*} \hat{u}_{8} \otimes \hat{\imath}^{*} \hat{u}_{8}+B_{3} \bar{\alpha} \neq 0,
$$


and there exists $\hat{u}_{15} \in H^{15}\left(B_{1}(\hat{X}), Z_{2}\right), \delta \hat{u}_{15}=w$ with

Observe that

$$
\delta: H^{*}\left(B_{1}(\hat{X}), \mathrm{Z}_{2}\right) \rightarrow H^{*}\left(B_{2}(\hat{X}), B_{1}(\hat{X}), \mathrm{Z}_{2}\right) .
$$

$$
0 \neq \Sigma^{*} u_{15} \in Q H^{14}\left(\hat{X}, \mathrm{Z}_{2}\right) / P H^{14}\left(\hat{X}, \mathrm{Z}_{2}\right)=Q H^{14}\left(X, \mathrm{Z}_{2}\right) / P H^{14}\left(X, \mathrm{Z}_{2}\right)
$$

by 3.2 , and the main theorem follows.

\section{REFERENCES}

1. J. F. Adams, On the non-existence of elements of Hopf invariant one, Ann. of Math. (2) 72 (1960), 20-104.

2. M. Curtis and G. Mislin, $H$-spaces which are bundles over $S^{7}$, J. Pure Appl. Algebra 1 (1971), 27-40.

3. S. Gitler and J. Milgram, Evaluating secondary operations on low dimensional classes, Conference on Algebraic Topology, University of Illinois, Chicago Circle 1968, 47-60.

4. P. Hilton and J. Roitberg, On principal $S^{3}$ bundles over spheres, Ann. of Math. (2) 90 (1969), 91-107.

5. P. Hilton and J. Roitberg, On the classification problem of $H$-spaces of rank 2, Comment. Math. Helv. 45 (1970), 506-516.

6. L. Kristensen, On secondary cohomology operations, Math. Scand. 12 (1963), 57-82.

7. J. Stasheff, Manifolds of the homotopy type of (non-Lie) groups, Bull. Amer. Math. Soc. 75 (1969), 998-1000.

8. A. Zabrodsky, Homotopy associativity and finite $C W$ complexes, Topology 9 (1970), 121-128.

9. A. Zabrodsky, Secondary operations in the cohomology of $H$-spaces, Illinois J. Math. 15 (1971), 648-655.

THE HEBREW UNIVERSITY OF JERUSALEM, ISRAEL 